media/PDFs/EUCAST_files/Breakpoint_tables/v_7.1_Breakpoint_ Tables.pdf. Accessed June 16, 2017.

11. Diancourt L, Passet V, Verhoef J, Grimont PA, Brisse S. Multilocus sequence typing of Klebsiella pneumoniae nosocomial isolates. J Clin Microbiol. 2005;43:4178-4182.

12. Brisse $\mathrm{S}$, Passet V, Haugaard AB, et al. wzi Gene sequencing, a rapid method for determination of capsular type for Klebsiella strains. J Clin Microbiol. 2013;51:4073-4078.

13. Sousa C, Novais Â, Magalhães A, Lopes J, Peixe L. Diverse high-risk B2 and D Escherichia coli clones depicted by Fourier transform infrared spectroscopy. Sci Rep 2013;3:3278.

14. Portaria no. 353-A/2013 de 4 de dezembro. Ministérios do Ambiente, Ordenamento do Território e Energia, da Saúde e da Solidariedade, Emprego e Segurança Social website. https://www.academiaadene.pt/download/pt/portaria-n-353-a2013recs-ventilacao-e-qai.pdf. Published 2013. Accessed June 16, 2017.

\section{Room Occupancy-Associated Transmission of MDRO, Clostridium difficile, or Norovirus: Results From a Room Surveillance Project}

To the Editor-Mitchell et $\mathrm{al}^{1}$ conducted a systematic review and meta-analysis to determine the risk of pathogen acquisition for patients associated with prior room occupancy. Overall, pooled acquisition odds ratio was 2.14 (95\% confidence interval [CI], 1.65-2.77) for study pathogens: methicillin-resistant Staphylococcus aureus (MRSA); vancomycin-resistant enterococci (VRE); Clostridium difficile; Acinetobacter spp; extendedspectrum $\beta$-lactamase-producing coliforms; Pseudomonas spp. When comparing data between gram-positive and gramnegative organisms, the pooled acquisition odds ratios were 2.65 (95\% CI, 2.02-3.47) for gram-negative organisms and 1.89 (95\% CI, 1.62-2.21) for gram-positive organisms. Most of the included studies were performed in areas highly endemic for multidrug-resistant organisms (MDROs).

In January 2016, we initiated a prospective room surveillance project to determine whether the room is a risk factor for transmission to future occupants in a tertiary-care center with a low endemic MDRO burden. In addition, we considered rooms occupied by patients with symptomatic $C$. difficile or norovirus infection with a presumed high level of environmental contamination, for which a virucidal and sporicidal disinfectant was used for terminal cleaning and disinfection. Only routine infection control quality assurance or mandatory reportable surveillance data were used for this study, which was conducted according to German Federal Infection Prevention Law (IfSG) and German Federal Data protection law (Bundesdatenschutzgesetz).

All positive microbiological results of MDROs, Clostridium difficile, and norovirus cases were reported to the infection control registry. A likely case or room occupancy-associated transmission was defined as an occurrence of the same organism in a patient in the same room in the 6 weeks following discharge of a patient with known colonization or infection. For this report, we analyzed our experience during the first year of surveillance:

We identified 130 MDRO-cases: 95 MRSA, 9 MDR Escherichia coli, 9 MDR Pseudomonas aeruginosa, 5 MDR Klebsiella pneumoniae, 5 MDR Serratia marcescens, 2 MDR Enterobacter cloacae, 2 VRE, 1 MDR Acinetobacter baumannii, and 1 MDR Citrobacter freundii. Among these, 20 cases were classified as nosocomial according to current German surveillance definitions (www.nrz-hygiene.de) and 19 of these were not related to prior room occupancy.

A single case of MDR Klebsiella pneumoniae met inclusion criteria and was related to a small outbreak in the neonatal intensive care unit with transmission by a suctioning device.

We reported 7 cases of norovirus and 39 patients with C. difficile to the registry. A single norovirus case was nosocomial and 7 C. difficile cases were nosocomial, but none was related to prior room occupancy.

In contrast to previous studies, ${ }^{2}$ we could not demonstrate an increased risk for newly admitted patients to a room that had been occupied by a patient with an MDRO, C. difficile, or norovirus. Thus, we conclude that routine terminal cleaning and disinfection of all reachable surfaces in the room is sufficient to prevent the spread of these organisms, even in cases with a presumed high level of environmental contamination (eg, vomiting and diarrhea). The only case primarily linked to a room was falsely positive; it was part of a point-source outbreak not related to room cleaning or disinfection.

Our study is limited by the low endemic rate of MDRO and the lack of compliance checks of terminal cleaning procedures, ${ }^{3,4}$ but it reflects realistic daily practice in a large tertiarycare center in Germany. Enhanced cleaning and disinfections strategies ${ }^{5}$ may therefore only be necessary if the room is identified as a risk factor for transmission within an institution by surveillance such as ours.

\section{ACKNOWLEDGMENTS}

Financial support: No financial support was provided relevant to this article.

Potential conflicts of interest: S.S.S. is shareholder and employee of the Deutsches Beratungszentrum für Hygiene (BZH GmbH).

\section{Sebastian Schulz-Stübner, MD, $\mathrm{PhD}{ }^{1}$ Peter Leonards, HFK; ${ }^{2}$ Petra Zimmer, HFK $^{2}$}

Affiliations: 1. Deutsches Beratungszentrum für Hygiene (BZH GmbH). Schnewlinstr. 10, 79098 Freiburg im Breisgau, Germany; 2. Klinikum Mutterhaus der Borromäerinnen, Feldstr. 16, 54290 Trier, Germany.

Address correspondence to Privatdozent Dr Sebastian Schulz-Stübner, Deutsches Beratungszentrum für Hygiene (BZH GmbH), Schnewlinstr. 10, 79098 Freiburg im Breisgau, Germany (Schulz-stuebner@bzh-freiburg.de). Infect Control Hosp Epidemiol 2017;38:1130-1131

(C) 2017 by The Society for Healthcare Epidemiology of America. All rights reserved. 0899-823X/2017/3809-0026. DOI: 10.1017/ice.2017.145 


\section{REFERENCES}

1. Mitchell BG, Dancer SJ, Anderson M, Dehn E. Risk of organism acquisition from prior room occupants: a systematic review and meta-analysis. J Hosp Infect 2015;91:211-217.

2. Huang SS, Datta R, Platt R. Risk of acquiring antibiotic-resistant bacteria from prior room occupants. Arch Intern Med 2006; 166:1945-1951.

3. Clifford R, Sparks M, Hosford E, et al. Correlating cleaning thoroughness with effectiveness and briefly intervening to affect cleaning outcomes: how clean is cleaned? PLoS One 2016;11: e0155779. doi: 10.1371/journal.pone.0155779.

4. Goodman ER, Platt R, Bass R, Onderdonk AB, Yokoe DS, Huang SS. Impact of an environmental cleaning intervention on the presence of methicillin-resistant Staphylococcus aureus and vancomycin-resistant enterococci on surfaces in intensive care unit rooms. Infect Control Hosp Epidemiol 2008;29:593-599.

5. Anderson DJ, Chen LF, Weber DJ, et al. Enhanced terminal room disinfection and acquisition and infection caused by multidrugresistant organisms and Clostridium difficile (the Benefits of Enhanced Terminal Room Disinfection study): a cluster-randomised, multicentre, crossover study. Lancet 2017;389:805-814.

\section{Clinical Care of Hematological Patients in a Bone Marrow Transplant Unit: Do Human Resources Influence Infection Incidence?}

To the Editor-Allogeneic stem cell transplantation (allo-SCT) is widely considered a curative option for many hematological malignancies. Bloodstream infections (BSIs) represent the most frequent infective event in allotransplanted patients, and their incidence may vary from $20 \%$ to $70 \% .{ }^{1-3}$ Prolonged neutropenia, gastrointestinal mucosal damage, and extensive use of central venous catheters (CVC) are the major risk factors for BSIs. ${ }^{4,5}$ Usually, BSIs occur during the pre-engraftment phase, but they can occur in later phases, too. ${ }^{4}$ Prophylactic antimicrobial therapy (namely with fluoroquinolones) is conventionally used during agranulocytosis, and empirical broadspectrum antibiotics (namely third-generation cephalosporins, aminoglycosides, and glycopeptides) are promptly started in the event of fever or suspected infection while waiting for the results of microbiological cultures to initiate a target therapy. ${ }^{6}$ Considering that only a minority of febrile episodes $(30 \%-40 \%)$ in patients subjected to allo-SCT can be defined as BSIs, surveillance for infections in a bone marrow transplant unit is mandatory, to correctly drive the use of empirical therapy.

We recently published the data on the incidence and outcome of BSIs in a cohort of 162 patients who had allo-SCT, over a period of 6 years of transplant activity. ${ }^{7}$ Briefly, $60 \%$ of the patients were transplanted for acute leukemias and $49 \%$ experienced a BSI, for a total of 119 isolates. The median time of blood culture positivity was 19 days from transplant (range day -4 to day +921$)$. Half $(n=59)$ of the positive cultures were from peripheral blood samples and half $(n=60)$ were central-venous-catheter related, defined as a positive catheter blood culture that preceded a positive peripheral blood sample by 2 hours. In 77 of 119 cases (65\%) and 42 of 119 cases (35\%), a gram-positive or a gram-negative agent were isolated, respectively. Staphylococcus epidermidis and Escherichia coli were the most common isolates ( $35 \%$ and $57 \%$, respectively). Concerning antimicrobial resistance, we observed fluoroquinolone resistance both among gram-positive (roughly $100 \%$ ) and gram-negative bacteria (between $90 \%$ and $100 \%$ ), together with methicillin resistance among gram-positive bacteria (100\% of the S. aureus, S. epidermidis, and S. haemolyticus, and $75 \%$ of $S$. hominis isolates). Moreover, $67 \%$ of E. coli were extended-spectrum $\beta$-lactamase producers, and $40 \%$ of Pseudomonas aeruginosa were resistant to carbapenems. Interestingly, no carbapenemase-producing Klebsiellae were isolated. Overall, 15 of 162 allotransplanted patients (9\%) died from BSIs. Pseudomonas aeruginosa is the most dangerous, with a $50 \%$ mortality rate. Enterococci, coagulase negative staphylococci, and E. coli showed mortality rates of $33 \%, 12 \%$, and $4 \%$, respectively.

The annual distribution of gram-positive and gram-negative bacteria and the gram-positive to gram-negative ratio are reported in Figure 1A and B. Within the 6-year observation period, we found a stable gram-positive to gram-negative ratio in all years except 2012 and 2013, when we observed a reduction in the number of positive blood cultures (11 in 2012 and 13 in 2013) and a reduction in the gram-positive to gram-negative ratio (1.2 in 2012 and 0.6 in 2013). Although these differences do not reach significance, they may be partially explained by different CVC management practices in 2012 and 2013, when we had a single dedicated nurse for CVC medications. Considering the high incidence of antibiotic resistance and the unavailability of prophylaxis other than fluoroquinolones, the adoption of clinical-care strategies, such as CVC medication given under optimal asepsis by dedicated nurses, may be the best way to prevent BSIs. This point is crucial, and we believe that the institution of a "CVC nursing team," trained to use the most stringent techniques for CVC management to limit the risk of CVC infection, may partially reduce the incidence of BSIs in allotransplanted patients. The problem, in daily clinical practice, is the lack of human resources to address this issue. Most Italian bone marrow transplant units suffer chronically from inadequate numbers of nurses to provide clinical care for patients. The clinical needs of allotransplanted patients during the first 4060 days following transplant are very similar to those reserved for patients admitted to intensive care units. In theory, 1 nurse per 3 patients is the ideal ratio. In our unit, we now have 1 nurse for every 6 patients. Consequently, some simple activities, such as CVC medication management, may not be conducted with optimal attention to asepsis.

In conclusion, BSIs are a significant event in allotransplanted patients; they can significantly influence morbidity and mortality 